Review

\title{
Genetic Risk of Psychosis in Relation to Cannabis Use: Findings from Polygenic Risk Score Approaches
}

\author{
Kristina Adorjan 1,2,*, Sergi Papiol 1,2 \\ 1 Institute of Psychiatric Phenomics and Genomics, Medical Center of the \\ University of Munich, Munich, 80336, Germany \\ 2 Department of Psychiatry and Psychotherapy, Medical Center of the University \\ of Munich, Munich, 80336, Germany \\ * Correspondence: Kristina Adorjan, \\ Email: kristina.adorjan@med.uni-muenchen.de; Tel.:+49-89-4400-55560.
}

\begin{abstract}
A psychotic disorder is a multifactorial phenomenon in which not only environmental influences but also genetic factors play an important role. Evidence indicates that psychotic disorders are characterized by a complex mode of inheritance with high polygenicity, in which a large number of common genetic variants with small effects are relevant. One way to measure this polygenic risk is the calculation of polygenic risk scores (PRS). These reflect the complex multifactorial interaction of coding and regulatory DNA variants in the development of mental illness. It is known that the use of cannabis in patients with schizophrenia (SCZ) is much higher than in the general population. Although an exact clinical prognosis based on PRS is not possible at the present, the results found by PRS investigations so far are quite promising. Initial results suggest that people with SCZ and an increased polygenic risk of schizophrenia are more likely to use cannabis. According to these results, the connection between mental illnesses and cannabis use could therefore not simply be seen as an environmental risk, but rather explained as a geneenvironment correlation.
\end{abstract}

\section{G Open Access}

Received: 14 January 2019

Accepted: 06 December 2019

Published: 11 December 2019

Copyright (c) 2019 by the author(s). Licensee Hapres, London, United Kingdom. This is an open access article distributed under the terms and conditions of Creative Commons Attribution 4.0 International License.
Keywords: schizophrenia; psychosis; cannabis; genetics; polygenic risk score

\section{INTRODUCTION}

Cannabis is the most widely used illicit drug in the world. The World Drug Report 2016 from the United Nations Office on Drugs and Crime reported that $3.8 \%$ of the global population used cannabis in 2015 [1]. While some cannabis users present a moderate to problematic use, a formal diagnosis of dependence according to DSM-V requires excessive use [2,3]. Although a direct causality between cannabis use and the development of psychiatric disorders has not been convincingly established, repeated use of cannabis has been associated with several 
medical conditions that include depression, psychosis and other mental health problems, physical health problems, and reduced quality of life [4].

It is well established that comorbid substance abuse, including cannabis abuse, is much more prevalent among patients with psychotic disorders than in the general population [5-8]. However, the relationship between cannabis use and psychosis might be more complicated than it appears at first. A psychotic disorder is a multifactorial phenomenon in which both environmental influences and genetic factors play an important role. Epidemiological studies have identified many environmental factors associated with an increased risk of psychosis, including childhood adversity, trauma, and viral infections [9]. Genetic studies have also revealed that a genetic predisposition to schizophrenia might be associated with increased use of cannabis in healthy individuals [10]. To date, however, no single genetic or environmental factor has been unambiguously identified as a direct cause of psychosis. The risk of developing psychosis increases with the accumulation of many genetic risk variants and exposure to several risk environmental factors [9].

In recent decades, the most important questions discussed in the field of cannabis research have been whether the use of cannabis increases the risk of psychosis and whether psychotic disorders occur more frequently in people who use cannabis [6-9]. The question has also arisen as to how widespread cannabis use and cannabis-related disorders are among people with a psychotic disorder.

An important body of evidence supports the notion that cannabis use, especially in adolescence, precedes the onset of psychosis [11]. This has been consistently reported in several longitudinal cohorts [12-16]. On the other hand, some studies have described the possibility that psychosis may lead to an increased propensity to cannabis use, and especially people with psychotic spectrum disorders are more likely to experiment with drugs [17-20].

This notion converges with the evidence of self-medication using cannabis in patients with psychotic disorders. Particularly negative symptoms can drive cannabis use in patients with schizophrenia which in turn may exacerbate positive symptoms [21].

Overall, researchers are interested to know whether and to what extent cannabis use affects the course and outcome of a psychotic disorder because of the implications of this information for treatment and the allocation of resources of the health system [22]. In relation to these questions, here we give an overview of the genetic risk of psychosis associated with cannabis use.

\section{EVIDENCE FROM CANDIDATE-GENE STUDIES}

In recent years, great progress has been made in the field of psychiatric genetics. Numerous association studies have been performed to identify genetic risk variants. Within the framework of candidate gene studies, 
studies first tested whether certain genetic markers (especially single nucleotide polymorphisms, SNPs) are associated with an increased risk of disease. Candidate genes were identified in the context of neurobiological processes underlying the psychosis development and the cannabinoid system, e.g., AKT1, CNR1, BDNF, and SLC6A4, suggesting a link between cannabis use and the development of a psychotic disorder [23]. Likewise, two further systematic reviews [24,25] reported an association but no clear evidence of a possible gene-environment interaction for psychosis and cannabis use. On the other hand, on the basis of comparatively small subsamples Ferretjans et al. [24] reported that a possible geneenvironment interaction may be associated with a genetic susceptibility of the endocannabinoid system. Some studies also suggested a role for the interaction of a functional variant in the COMT gene with psychometric psychosis liability and cannabis exposure on the emergence of psychotic experiences [26]. Because of the small sample sizes in candidate gene studies, however, only a few replicable results could be obtained. Large case-control studies have shown that these variants have minor effects (usually $\mathrm{OR}<1.3$ ), suggesting that detecting such gene $\times$ environment interactions may also require large sample sizes. Many candidate gene studies have focused on a few polymorphisms (mostly SNPs) in coding regions of the DNA. However, current evidence indicates that a large proportion of variants associated with complex polygenic diseases are often found in regulatory regions, introns or intragenic regions [27,28].

\section{EVIDENCE FROM GENOME-WIDE ASSOCIATION STUDIES}

The decisive step towards identifying risk alleles was taken with genome-wide association studies (GWAS). In these studies, large numbers of SNPs are used to test as many regions of the genome as possible for associations with disease risk. $P$ values below $5 \times 10^{-8}$ are considered to indicate genome-wide significance. As a result of the collaboration of international consortia, the number of cases available for GWAS is continuously increasing. Within the framework of the Psychiatric Genomics Consortium (PGC), GWAS based on tens to hundreds of thousands of patients and controls have led for the first time to the discovery of replicable markers and have provided evidence of the polygenicity of psychiatric disorders and related traits [29]. Likewise, large GWAS using cannabis use as target phenotype have already identified specific loci associated with this trait (highlighting CADM2 and NCAM1, among other genes) and have also showed evidence of the polygenicity of this trait [17,30,31].

In this context, the results of GWAS now allow the study of the genetic correlation between cannabis use and psychosis as well as their bidirectional association. Gage et al., using bidirectional two-sample mendelian randomization, found strong evidence that schizophrenia risk predicts cannabis initiation, and some weaker evidence that cannabis use predicts increased risk of schizophrenia [17]. Vaucher et al. performed a 
similar analysis using cannabis use as target phenotype, and found evidence that cannabis use was associated with an increased risk of schizophrenia [30]. The most recent and largest GWAS on lifetime cannabis use $(N=184,765)$ found evidence of a genetic correlation between schizophrenia and cannabis use $\left(r_{\mathrm{g}}=0.2452\right)$ and, in line with Gage et al. but in contrast to Vaucher et al., strong evidence for a causal positive influence of schizophrenia risk on lifetime cannabis use but not the other way around [31].

\section{POLYGENICITY}

In addition to the genome-wide significant associations discovered to date, numerous markers have been identified whose influence does not reach genome-wide significance. Even though these markers have no direct association with the phenotype (the association may not be strong enough to pass genome-wide significance thresholds), they can be grouped together, representing the polygenic signature of a disease. These markers, when considered en masse, can explain a larger proportion of phenotypic variance than a score derived of only genome-wide significant variants [29]. The strength of the polygenic association depends, to a large extent, on the power of the original "discovery sample" from which the allelic risk information is derived; this in turn correlates with the sample size. Within this framework, so called polygenic risk scores (PRS) can be calculated for phenotype prediction. Briefly, from the results of a given GWAS ("training set"), PRS can be calculated for individuals in an independent sample on the basis of the identified risk allele and effect size for each SNP. In such analyses, a score is calculated for the subjects of the independent replication sample by forming a weighted sum of the associated risk alleles of each individual [29].

PRS are of special research interest as a genetic tool that summarizes individual genetic load. Statistical analyses using PRS as a "composite genetic risk value" have shown that they are able to explain a moderate amount of phenotypic variance of the target phenotype (e.g., schizophrenia) and, in contrast to a vast majority of candidate-gene analyses, they show a very good replicability across different independent test samples. On the basis of PRS analyses, Power et al. have found that healthy individuals with an increased genetic predisposition to schizophrenia consume cannabis more frequently and in larger amounts than individuals with lower genetic risk scores (PRS for schizophrenia showed positive associations for "ever" $v s$ "never" using cannabis across all $P$-value thresholds; the strongest association for SNPs with $P<0.01$ in original schizophrenia GWAS-[PGC] was $R^{2}=0.47 \%, P=2.6 \times 10^{-4}$ ). Significant associations were also observed in the analysis of the amount of cannabis for 9 of the $10 P$-value cutoffs; the strongest association was found for the SNPs with $P \leq 0.05$ for schizophrenia $\left(R^{2}=0.85 \%, P=0.003\right)$ [10]. The results of this study and from some of the aforementioned mendelian randomization studies suggest a gene-environment correlation due to the 
fact that subjects seem to define their environment based on their innate preferences.

Similar results were obtained in recent independent studies. Individuals with a genetic predisposition to schizophrenia consumed cannabis more frequently and more often throughout their lives than individuals with a lower genetic burden (PRS for schizophrenia were significant $(P<0.05)$ when five of the eight cannabis use phenotypes were tested in the target sample, including lifetime use, regular use, and amount consumed, with risk scores explaining up to $0.5 \%$ of the variance) [32]. Moreover, recent research from our group has suggested a positive association between PRS for schizophrenia in patients with bipolar disorder (BD): PRS for schizophrenia showed positive associations for "ever" vs "never" using cannabis in BD across most $P$-value thresholds, with an $R^{2}$ of $1 \%$. These unpublished results could be replicated in an independent sample [33].

Results in a large Icelandic cohort $(N=144,609)$ have shown a similar effect of polygenic risk for SCZ (and to a lesser extent polygenic BD risk) being associated not only with cannabis use disorder but also with other substance use disorders and smoking [34]. Specifically, in this study a high SCZ polygenic load was associated with an increased risk of cannabis use disorder (OR $=1.23 ; 95 \%$ CI: 1.18-1.29).A recent study in adolescent population has shown a similar trend regarding cannabis use, with SCZ PRS associated with a stronger increase in cannabis use at age 16-20 [35]. However, this study was not able to find an association between SCZ PRS and alcohol us or smoking.

A recent report from the EUGEI study has shed light on the interplay between SCZ PRS and cannabis use (and other environmental factors) [36]. This study shows evidence for additive effects (interaction) of cannabis use on the association between the polygenic risk score for schizophrenia and SCZ-control status (relative excess risk due to interaction $(\mathrm{RERI})=5.60$; 95\% CI: 0.88-10.33). No evidence for gene-environment correlation between SCZ-PRS and cannabis use (OR $=0.98$; 95\% CI: $0.61-1.59$ ) was found in this study.

\section{CONCLUSION}

In summary, the question of the role of cannabis use in the development of psychotic disorders cannot be answered conclusively. Epidemiological studies indicate that the strength of the link between cannabis use and psychosis should be revised, taking into account other factors that are also associated with an increased risk of psychosis. Moore et al. tried to correct these factors and found a 1.4- to 2.1-times higher risk of developing psychosis through cannabis use [8]. In addition, several studies have shown that cannabis effects are independent of alcohol consumption or the use of other drugs [12-16].

PRS analyses can be used to investigate genetic effects with respect to clinically relevant phenomena. Although at present a precise clinical 
prognosis is not possible on the basis of PRS, the results found in PRS studies to date suggest that they could be part of future predictive models including clinical and biological features. Initial results suggest a transdiagnostic effect across psychiatric patients and healthy subjects with an increased polygenic risk for schizophrenia associated with cannabis use/cannabis use disorder. The connection between mental illness and cannabis use may therefore not simply be an environmental risk, but rather the result of a gene-environment correlation and/or interaction. In the future, larger sample sizes will be necessary to investigate the genetic association between a mental disorder and cannabis use and to identify common genes and biological mechanisms that may explain this association. Moreover, in a different but complementary approach, the enormous technical progress in the field of genomic high-throughput technologies (next generation sequencing) will probably lead to new insights into the influence of rare variants (low frequency) in the coming years; first molecular genetic studies have already shown that common and rare variants contribute to the genetic risk for psychosis [18].

\section{CONFLICTS OF INTEREST}

The authors declare that they have no conflicts of interest.

\section{REFERENCES}

1. United Nations Office on Drugs and Crime. World Drug Report. New York (US): United Nations on Drugs and Crime; 2016. Available from: https://www.unodc.org/wdr2016/. Accessed 2019 Dec 6.

2. Hasin DS, Saha TD, Kerridge BT, Goldstein RB, Chou SP, Zhang H, et al. Prevalence of marijuana use disorders in the United States between 20012002 and 2012-2013. JAMA Psychiatry. 2015;72:1235-42.

3. American Psychiatric Association. Diagnostic and Statistical Manual of Mental Disorders (DSM-5). Washington, D.C. (US): American Psychiatric Association; 2013.

4. Lovell ME, Bruno R, Johnston J, Matthews A, McGregor I, Allsop DJ, et al. Cognitive, physical, and mental health outcomes between long-term cannabis and tobacco users. Addict Behav. 2018;79:178-88.

5. Kraan T, Velthorst E, Koenders L, Zwaart K, Ising HK, van den Berg D, et al. Cannabis use and transition to psychosis in individuals at ultra-high risk: Review and meta-analysis. Psychol Med. 2015;46(4): 673-81.

6. Linscott RJ, van Os J. An updated and conservative systematic review and meta-analysis of epidemiological evidence on psychotic experiences in children and adults: On the pathway from proneness to persistence to dimensional expression across mental disorders. Psychol Med. 2013;43(6):1133-49.

7. Marconi A, Di Forti M, Lewis CM, Murray RM, Vassos E. Meta-analysis of the association between the level of cannabis use and risk of psychosis. Schizophr Bull. 2016;42(5):1262-9. doi: 10.1093/schbul/sbw003 
8. Moore TH, Zammit S, Lingford-Hughes A, Barnes TR, Jones PB, Burke M, et al. Cannabis use and risk of psychotic or affective mental health outcomes: a systematic review. Lancet. 2007;370(9584):319-28.

9. van Os J. Krabbendam L, Myin-Germeys I, Delespaul P. The schizophrenia envirome. Curr Opin Psychiatry. 2005;18(2):141-5.

10. Power RA, Verweij KJ, Zuhair M, Montgomery GW, Henders AK, Heath AC, et al. Genetic predisposition to schizophrenia associated with increased use of cannabis. Mol Psychiatry. 2014;19(11):1201-4. doi: 10.1038/mp.2014.51

11. Volkow ND, Swanson JM, Evins AE, DeLisi LE, Meier MH, Gonzalez R, et al. Effects of Cannabis Use on Human Behavior, Including Cognition, Motivation, and Psychosis: A Review. JAMA Psychiatry. 2016;73(3):292-7.

12. Arseneault L Cannon M, Poulton R, Murray R, Caspi A, Moffitt TE. Cannabis use in adolescence and risk for adult psychosis: longitudinal prospective study. BMJ. 2002;325(7374):1212-3.

13. Andréasson S, Allebeck P, Engström A, Rydberg U. Cannabis and schizophrenia: a longitudinal study of Swedish conscripts. Lancet. 1987;2(8574):1483-6.

14. Zammit S, Allebeck P, Andreasson S, Lundberg I, Lewis G. Self reported cannabis use as a risk factor for schizophrenia in Swedish conscripts of 1969: historical cohort study. BMJ. 2002;325(7374):1199.

15. van Os J, Bak M, Hanssen M, Bijl RV, de Graaf R, Verdoux H. Cannabis use and psychosis: a longitudinal population-based study. Am J Epidemiol. 2002;156(4):319-27.

16. Fergusson DM, Horwood LJ, Swain-Campbell NR. Cannabis dependence and psychotic symptoms in young people. Psychol Med. 2003;33(1):15-21.

17. Gage SH, Jones HJ, Burgess S, Bowden J, Davey Smith G, Zammit S, et al. Assessing causality in associations between cannabis use and schizophrenia risk: a two-sample Mendelian randomization study. Psychol Med. 2017;47(5):971-80.

18. Zwicker A, Denovan-Wright EM, Uher R. Gene-environment interplay in the etiology of psychosis. Psychol Med. 2018;48(12):1925-36.

19. Ferdinand RF, Sondeijker F, van der Ende J, Selten JP, Huizink A, Verhulst FC. Cannabis use predicts future psychotic symptoms, and vice versa. Addiction. 2005;100(5):612-8.

20. Foti DJ, Kotov R, Guey LT, Bromet EJ. Cannabis use and the course of schizophrenia. 10-year follow-up after first hospitalization. Am J Psychiatry. 2010;167:987-93.

21. Gill KE, Poe L, Azimov N, Ben-David S, Vadhan NP, Girgis R, et al. Reasons for cannabis use among youths at ultra high risk for psychosis. Early Interv Psychiatry. 2015;9(3):207-10.

22. Hoch E, Friemel CM, Schneider M, editors. Cannabis Expertise. Cannabis: Potenzial und Risiko. Eine wissenschaftliche Bestandsaufnahme. Basel (Switzerland): Springer Verlag; 2018. p.189-213. German.

23. Uliana V, Tomassini A, Pollice R, Gennarelli M, Faravelli F, Casacchia M, et al. Cannabis and psychosis: A systematic review of genetic studies. Curr Psychiat Rev. 2013;9(4):302-15. 
24. Ferretjans R, Moreira FA, Teixeira AL, Salgado JV. The endocannabinoid system and its role in schizophrenia: A systematic review of the literature. Braz J Psychiatry. 2012;34(Suppl 2):S163-93.

25. Geoffroy PA, Etain B, Houenou J. Gene $\times$ environment interactions in schizophrenia and bipolar disorder: Evidence from neuroimaging. Front Psychiatry. 2013;4:136. doi: 10.3389/fpsyt.2013.00136

26. Henquet C, Rosa A, Krabbendam L, Papiol S, Fananás L, Drukker M, et al. An experimental study of catechol-o-methyltransferase Val158Met moderation of delta-9-tetrahydrocannabinol-induced effects on psychosis and cognition. Neuropsychopharmacology. 2006;31(12):2748-57.

27. Zondervan KT. Genetic Association Study Design. In: Zeggini E, Morris A, editors. Analysis of Complex Disease Association Studies. Burlington (US): Elsevier; 2011. p. 25-47.

28. Goes FS. Genetics of Bipolar Disorder: Recent Update and Future Directions. Psychiatr Clin North Am. 2016;39(1):139-55. doi: 10.1016/j.psc.2015.10.004

29. Maier RM, Visscher PM, Robinson MR, Wray NR. Embracing polygenicity: a review of methods and tools for psychiatric genetics research. Psychol Med. 2018;48(7):1055-67.

30. Vaucher J, Keating BJ, Lasserre AM, Gan W, Lyall DM, Ward J, et al. Cannabis use and risk of schizophrenia: a Mendelian randomization study. Mol Psychiatry. 2018;23:1287-92.

31. Pasman JA, Verweij KJH, Gerring Z, Stringer S, Sanchez-Roige S, Treur JL, et al. GWAS of lifetime cannabis use reveals new risk loci, genetic overlap with psychiatric traits, and a causal influence of schizophrenia. Nat Neurosci. 2018;21(9):1161-70.

32. Verweij K, Abdellaoui A, Nivard MG, Sainz Cort A, Ligthart L, Draisma HH, et al. Short communication: Genetic association between schizophrenia and cannabis use. Drug Alcohol Depend. 2017;171:117-21. doi: 10.1016/j.drugalcdep.2016.09.022

33. Adorjan K, Lütticke A, Budde M, Heilbronner U, Schulze TG, Papiol S, et al. Polygenic risk burden and cannabis use comorbidity in patients with schizophrenia and bipolar disorder. 2019. Unpublished work.

34. Reginsson GW, Ingason A, Euesden J, Bjornsdottir G, Olafsson S, Sigurdsson E, et al. Polygenic risk scores for schizophrenia and bipolar disorder associate with addiction. Addict Biol. 2018;23(1):485-92. doi: 10.1111/adb.12496

35. Hiemstra M, Nelemans SA, Branje S, van Eijk KR, Hottenga JJ, Vinkers CH, et al. Genetic vulnerability to schizophrenia is associated with cannabis use patterns during adolescence. Drug Alcohol Depend. 2018;190:143-50.

36. Guloksuz S, Pries LK, Delespaul P, Kenis G, Luykx JJ, Lin BD, et al. Examining the independent and joint effects of molecular genetic liability and environmental exposures in schizophrenia: results from the EUGEI study. World Psychiatry. 2019;18(2):173-82.

How to cite this article:

Adorjan K, Papiol S. Genetic Risk of Psychosis in Relation to Cannabis Use: Findings from Polygenic Risk Score Approaches. J Psychiatry Brain Sci. 2019;4:e190018. https://doi.org/10.20900/jpbs.20190018 